\title{
Comparative risk of malignancies and infections in patients with rheumatoid arthritis initiating abatacept versus other biologics: a multi-database real-world study
}

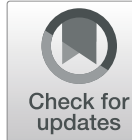

\author{
Teresa A. Simon ${ }^{1 *}$ (D, Marten Boers ${ }^{2}$, Marc Hochberg ${ }^{3}$, Nicole Baker ${ }^{1}$, Mary L. Skovron ${ }^{1}$, Nitesh Ray ${ }^{4}$,
} Sanket Singhal ${ }^{4}$, Samy Suissa ${ }^{5}$ and Andres Gomez-Caminero ${ }^{1}$

\begin{abstract}
Background: Patients with rheumatoid arthritis (RA) are at an increased risk of developing certain cancers and infections compared with the general population. Biologic and targeted synthetic disease-modifying antirheumatic drugs (b/tsDMARDs) are effective treatment options for RA, but limited evidence is available on the comparative risks among b/tsDMARDs. We assessed the risk of malignancies and infections in patients with RA who initiated abatacept versus other b/tsDMARDs in a real-world setting.
\end{abstract}

Methods: This retrospective, observational study used administrative data from three large US healthcare databases (MarketScan, PharMetrics, and Optum) to identify patients treated with abatacept or other b/tsDMARDs. In both groups, age-stratified incidence rates (IRs) with 95\% confidence intervals (Cls) were calculated for total malignancy and hospitalized infections; propensity score matching and Cox proportional hazards regression models were used to estimate hazard ratios (HRs) with 95\% Cls for total malignancy, lung cancer, lymphoma, breast cancer, nonmelanoma skin cancer (NMSC), hospitalized infections, opportunistic infections, and tuberculosis (TB), both within individual databases and in meta-analyses across the three databases.

Results: A rounded total of 19.2, 13.6, and 4.2 thousand patients initiating abatacept and 55.3, 40.8, and 13.8 thousand initiating other b/tsDMARDs were identified in the MarketScan, PharMetrics, and Optum databases, respectively. The IRs for total malignancy and hospitalized infections were similar between the two groups in each age stratum. In meta-analyses, total malignancy risk (HR [95\% CI] 1.09 [1.02-1.16]) of abatacept versus other b/ tsDMARDs was slightly but statistically significantly increased; small, but not statistically significant, increases were seen for lung cancer (1.10 [0.62-1.96]), lymphoma (1.27 [0.94-1.72]), breast cancer (1.15 [0.92-1.45]), and NMSC (1.10 [0.93-1.30]). No significant increase in hospitalized infections (0.96 [0.84-1.09]) or opportunistic infections (1.06 [0.96-1.17]) was seen. For TB, low event counts precluded meta-analysis.

Conclusions: In this real-world multi-database study, the risks for specific cancers and infections did not differ significantly between patients in the abatacept and other b/tsDMARDs groups. The slight increase in total malignancy risk associated with abatacept needs further investigation. These results are consistent with the established safety profile of abatacept.

Keywords: Abatacept, Real-world data, Claims, Infections, Malignancies, Rheumatoid arthritis

\footnotetext{
* Correspondence: teresa.simon1@verizon.net

Author's Teresa A. Simon and Nicole Baker are both affiliated with Bristol-Myers

Squibb, Princeton, NJ, USA at the time of the analysis.

${ }^{1}$ Bristol-Myers Squibb, Princeton, NJ, USA

Full list of author information is available at the end of the article
}

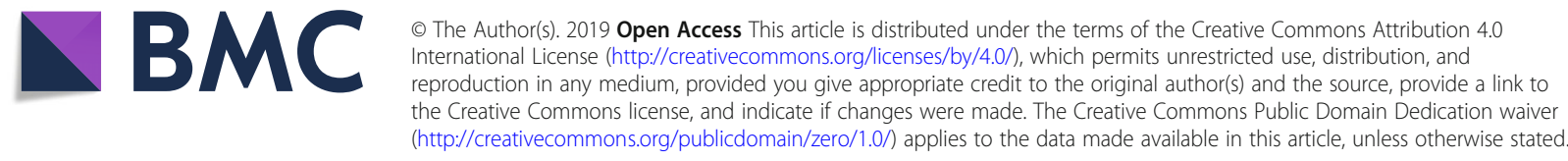




\section{Introduction}

Patients with rheumatoid arthritis (RA) not only bear a high burden of disease [1-4], but are also at an increased risk of developing infections and certain cancers, particularly lymphomas and lung cancer, compared with the general population [5-9]. Given the chronic nature of RA and the requirement for prolonged treatment, it is important for physicians to consider the long-term safety of different treatments in addition to their efficacy when making treatment decisions.

Biologic and targeted synthetic disease-modifying antirheumatic drugs (b/tsDMARDs) are effective treatments for RA. However, current evidence is inconsistent as to whether the use of $b /$ tsDMARDs generally or specific $b$ / tsDMARDs increases the risk of malignancies and infections in the RA population. Higher rates of malignancies [10] and infections $[10,11]$ have been observed with tumor necrosis factor (TNF) inhibitors when compared with placebo or conventional synthetic (cs) DMARDs in some studies. Other studies either showed no increased risk with bDMARDs versus csDMARDs [12-15] or were inconclusive [16]. When safety risks across different bDMARDs were examined, similar rates of malignancies and infections with abatacept and adalimumab were reported in a phase III randomized clinical trial [17]. Furthermore, a systematic literature review informing the 2016 update of the European League Against Rheumatism recommendations recognized a lack of evidence on the risks of malignancies, tuberculosis (TB), and herpes zoster virus across bDMARDs [18].

Abatacept, a selective $\mathrm{T}$ cell co-stimulation modulator approved for the treatment of RA, has a mechanism of action that is fundamentally different from that of other b/tsDMARDs [19-22]. Several clinical trials have demonstrated the efficacy, tolerability, and safety profile of abatacept [23-26]. Low incidence rates (IRs) for malignancies and infections have been reported with abatacept treatment in clinical trials [14, 23, 26, 27]; however, the risks associated with the long-term use of abatacept versus other $\mathrm{b} / \mathrm{tsDMARDs}$ in the real-world setting need further investigation [28]. This study used information from three large US healthcare claims databases to evaluate the risk of malignancies and infections in patients with RA treated with either abatacept or other b/ tsDMARDs in a real-world setting.

\section{Methods}

\section{Study design and data sources}

Data from three US administrative healthcare databases were included in this retrospective, real-world, observational study: Truven MarketScan Commercial and Supplemental Medicare (MarketScan), IMS PharMetrics (PharMetrics), and Optum Clinformatics Data Mart (Optum). As of September 2014, the MarketScan database had information on over 70 million privately insured patients aged under 65 years and approximately 6 million patients aged 65 years or older receiving Medicare coverage from 2006 onwards. The PharMetrics database contains claims information on approximately 55 million patients from over 90 managed care plans and other sources starting from 2007. The Optum database contains claims information and enrollment data on approximately 35 million patients from 2002 onwards. Available data in all three databases included demographic variables, diagnostic codes, and treatment information.

\section{Patient population}

Adult patients (aged $\geq 18$ years) with RA who initiated abatacept or other b/tsDMARD treatments (index date) from July 1, 2006, to September 30, 2014, and had 180 or more days of continuous health plan enrollment before the index date were eligible for inclusion. Identification of patients with RA was based on MacLean's criteria [29], which requires two or more inpatient or outpatient International Classification of Diseases, Ninth Revision, Clinical Modification (ICD-9-CM) diagnosis codes for RA (714.xx) in the patient's history (prior to and including the index date) or within 6 months of the index date. Patients were assumed to have initiated a treatment when there was no claim for that particular drug in the 180-day period before the index date. Thus, patients in this study could be first-time initiators for that drug, could have stopped that treatment $>180$ days before the index date and subsequently restarted, or could be switching from another b/tsDMARD. Patients who initiated abatacept at any point during the study period were included in the abatacept group; those who initiated more than one b/tsDMARD (excluding abatacept) were assigned to the first drug initiated. Treatments were identified using national drug codes for dispensed medications and procedure codes for injection or infusion. Other b/tsDMARDs were infliximab, etanercept, adalimumab, certolizumab pegol, golimumab, tocilizumab, rituximab, tofacitinib, and anakinra.

\section{Study outcomes}

All patients identified for the abatacept and other b/ tsDMARDs groups were included in the analyses within each database and meta-analyses across all three databases. ICD-9-CM diagnosis codes were used to identify the safety outcomes investigated: total malignancy (including non-melanoma skin cancer [NMSC]), lung cancer, lymphoma, breast cancer, NMSC, hospitalized infections, opportunistic infections, and TB. For each outcome computation, patients who had at least one ICD-9-CM code for that outcome in the 6-month period before the index date were excluded from the analysis of that particular outcome. 
All malignancy outcomes were identified by ICD-9CM codes. The follow-up period extended from 180 days after the index date until the occurrence of a malignancy, end of enrollment in the database, or end of data collection, whichever occurred first. A latency period of 180 days was included to allow for plausible induction time for the appearance of a malignancy associated with a new treatment and to avoid protopathic bias. The latency period and any malignancies that occurred during the latency period were not included in the analysis of malignancy outcomes.

For infection-related outcomes, the follow-up period extended from the index date to the occurrence of the infection of interest, end of the initiated treatment plus 90 days, end of enrollment in the database, or end of data collection, whichever occurred first. Infection-related outcomes were identified using ICD-9-CM diagnosis codes in conjunction with an inpatient stay or an outpatient physician visit procedure code. Opportunistic infections were identified using ICD-9-CM codes for primary TB infection, TB, herpes zoster virus, dermatophytosis, dermatomycosis, candidiasis, coccidioidomycosis, histoplasmosis, blastomycosis, and opportunistic or other mycoses. Classification of a case of $\mathrm{TB}$ required (1) prescription of a medication regimen including pyrazinamide, (2) an ICD9-CM code for TB and prescriptions for two anti-TB medications excluding pyrazinamide, or (3) an ICD-9-CM code for TB and an order for a TB diagnostic test [30].

\section{Statistical analyses}

Descriptive statistics were used to analyze baseline demographic information, co-morbid conditions, and concomitant medication use. For each outcome of interest, the IR and hazard ratio (HR), with 95\% confidence intervals (CIs), were calculated. IRs were calculated within each individual database by dividing the number of events for each outcome by the total person-time at risk, stratified by age group (18-64, 65-74, and $\geq 75$ years). A Poisson distribution was assumed to compute 95\% CIs for IRs.

To compare the safety events of abatacept with those of other b/tsDMARDs, HRs with 95\% CIs were calculated using multivariate Cox proportional hazards regression models. The calculations were performed separately for each individual database and across all three databases as a meta-analysis according to published methods [31-33]. Two methods were used to control for confounding factors: a propensity score-matched analysis and a propensity score-adjusted analysis. A propensity score was developed for each patient by index date within each database. Propensity scores were estimated separately within each database by logistic regression analyses incorporating measured potential predictors of therapy (Additional file 1: Tables S1-S6) as independent variables in the regression model and comparison group status as the outcome. In the matched analyses, each patient in the abatacept group was matched to up to two patients from the comparison group to achieve a balance between comparison groups in terms of all identified predictors of abatacept initiation. Propensity score matching was used for the analysis of all outcomes except TB. For the analysis of the outcome of $\mathrm{TB}$, a published algorithm developed by Calderwood et al. [30] was applied to the propensity score-adjusted model in which the variable imbalances between the groups were examined using Cohen's $d$ test and adjusted for in the final outcome model. The propensity score-adjusted model was used instead of the propensity score matching model to maximize the number of patients in the TB analysis. Additional details regarding the propensity scorematched and propensity score-adjusted analyses can be found in Additional file 1 (statistical analyses: variable selection for models).

To validate the level of specificity for the outcome identification, a sensitivity analysis was performed in which two ICD-9-CM codes for opportunistic infections were required in order to be counted as an event. The date of the first ICD-9-CM code claim was used as the event date for computation.

\section{Results}

\section{Patient disposition}

A rounded total of 19.2, 13.6, and 4.2 thousand patients initiating abatacept and 55.3, 40.8, and 13.8 thousand initiating other $\mathrm{b} /$ tsDMARDs were identified in the MarketScan, PharMetrics, and Optum databases, respectively (Fig. 1; Additional file 1: Table S7). After matching, 17.5, 12.1, and 3.4 thousand patients initiating abatacept and 32.3, 21.1, and 5.6 thousand initiating other b/ tsDMARDs were included from the MarketScan, PharMetrics, and Optum databases, respectively (Table 1).

\section{Baseline characteristics}

Baseline characteristics were generally well balanced across both groups (Additional file 1: Table S7). However, in each database, patients in the abatacept group were slightly older, more likely to be female, less likely to have another autoimmune disease, and more likely to have had exposure to b/tsDMARDs and csDMARDs at baseline (within 180 days before the index date) compared with those in the other $\mathrm{b} /$ tsDMARDs group (Additional file 1: Table S7). Patients were also more likely to have had a previous claim for hospitalized infections or cardiovascular disease in the abatacept group than those in the other b/tsDMARDs group. Distribution of index treatments among patients in the b/tsDMARDs group was similar across the three databases, with TNF inhibitors being the most commonly used (Additional file 1: 


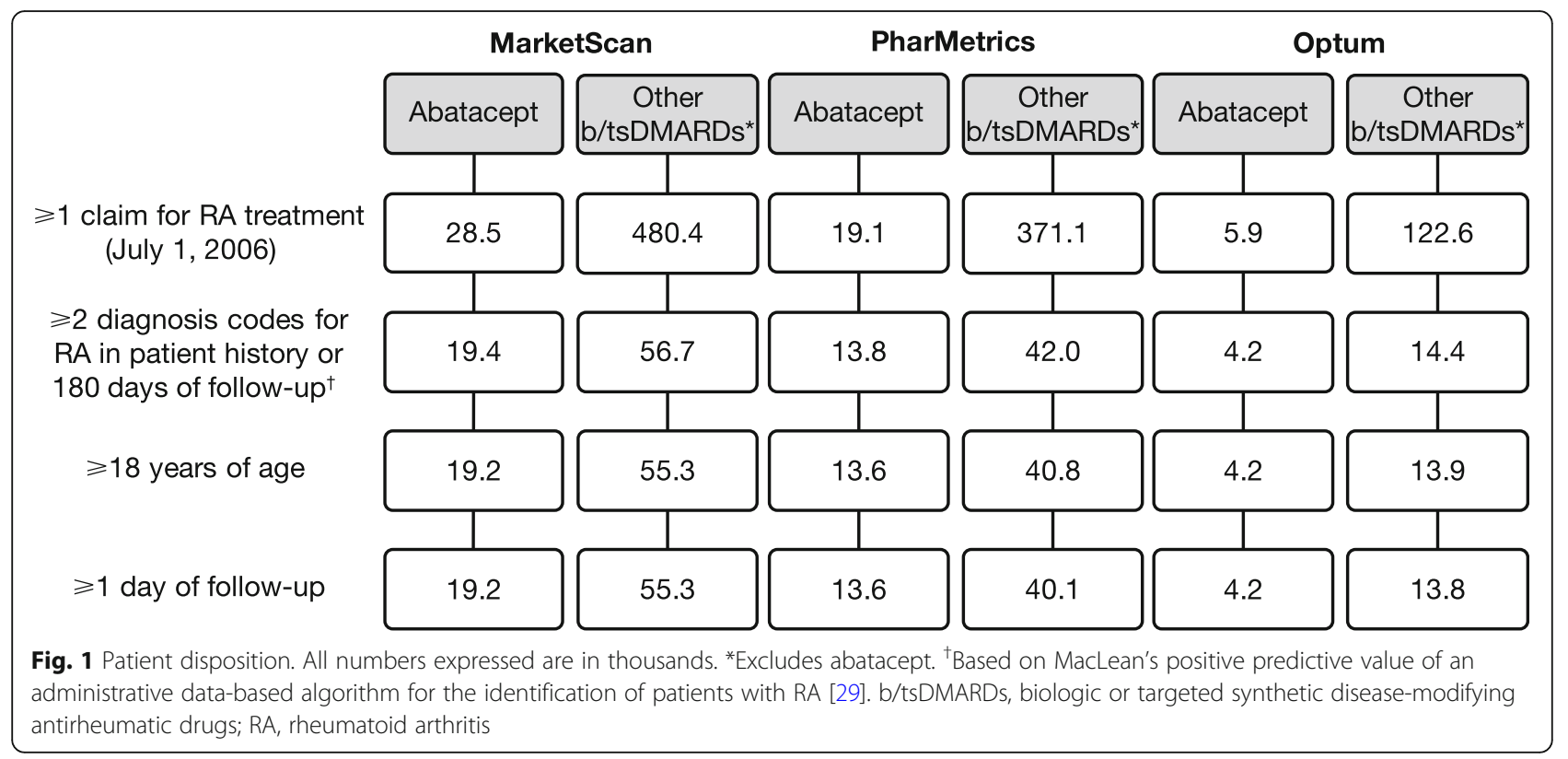

Table S8). In matched patients, all demographics and baseline characteristics were comparable except for exposure to b/tsDMARDs at baseline (within 180 days before the index date), which was greater in the abatacept group compared with the other b/tsDMARD group (Table 1).

\section{Outcomes}

\section{Malignancy outcomes}

The IRs for total malignancy were slightly higher in the abatacept-treated patients relative to the other b/ tsDMARDs group in each age stratum (Additional file 1: Table S9). The regression analyses in individual databases

Table 1 Demographics and baseline characteristics of patients in the matched abatacept and other b/tsDMARDs groups

\begin{tabular}{|c|c|c|c|c|c|c|}
\hline & \multicolumn{2}{|l|}{ MarketScan } & \multicolumn{2}{|l|}{ PharMetrics } & \multicolumn{2}{|l|}{ Optum } \\
\hline & $\begin{array}{l}\text { Abatacept } \\
(n=17,517)\end{array}$ & $\begin{array}{l}\text { Other b/tsDMARDs* } \\
(n=32,277)\end{array}$ & $\begin{array}{l}\text { Abatacept } \\
(n=12,120)\end{array}$ & $\begin{array}{l}\text { Other b/tsDMARDs* } \\
(n=21,145)\end{array}$ & $\begin{array}{l}\text { Abatacept } \\
(n=3354)\end{array}$ & $\begin{array}{l}\text { Other b/tsDMARDs* } \\
(n=5604)\end{array}$ \\
\hline Female, \% & 82 & 83 & 80 & 81 & 82 & 82 \\
\hline Age in years at index date, mean (SD) & $55(13)$ & $54(13)$ & $53(12)$ & $52(12)$ & $51(11)$ & $51(11)$ \\
\hline \multicolumn{7}{|c|}{ Co-morbid conditions during the baseline period, $\%$} \\
\hline Malignancy & 4.8 & 4.2 & 4.5 & 3.7 & 3.0 & 1.9 \\
\hline Cardiovascular disease $^{\dagger}$ & 22 & 19 & 21 & 17 & 21 & 17 \\
\hline Hospitalized infections & 3.2 & 2.3 & 3.5 & 2.6 & 3.7 & 2.9 \\
\hline Other autoimmune diseases ${ }^{\ddagger}$ & 17 & 15 & 20 & 18 & 17 & 16 \\
\hline \multicolumn{7}{|l|}{ Co-medications ${ }^{\S}, \%$} \\
\hline csDMARDs & 56 & 53 & 62 & 58 & 66 & 61 \\
\hline $\mathrm{b} / \mathrm{ts} \mathrm{DMARDs}$ & 48 & 15 & 53 & 17 & 59 & 21 \\
\hline Glucocorticoids & 54 & 53 & 61 & 59 & 71 & 71 \\
\hline
\end{tabular}

b/tsDMARDs biologic or targeted synthetic disease-modifying antirheumatic drugs, cSDMARDs conventional synthetic disease-modifying antirheumatic drugs, $S D$ standard deviation

*Excludes abatacept

${ }^{\dagger}$ Includes ischemic heart disease; diseases of pulmonary circulation; other forms of heart disease; cerebrovascular disease; diseases of the arteries, arterioles, and capillaries; diseases of the veins and lymphatics; and other diseases of the circulatory system

${ }^{\ddagger}$ Includes psoriatic arthropathy, other psoriasis, diabetes mellitus, multiple sclerosis, systemic lupus erythematosus, vitiligo, toxic diffuse goiter without mention of thryrotoxic crisis or storm, chronic lymphocytic thyroiditis, corticoadrenal insufficiency, acquired hemolytic anemias, immune thrombocytopenic purpura, chronic glomerulonephritis, cirrhosis of liver without mention of alcohol, celiac disease, regional enteritis, ulcerative enterocolitis, postinflammatory pulmonary fibrosis, giant cell arteritis, sicca syndrome, systemic sclerosis, alopecia areata, and urticaria

${ }^{s}$ Includes medications taken within 180 days before the index date 
showed no significant difference in the risk of total malignancy in abatacept initiators compared with other b/ tsDMARDs initiators (Fig. 2a), although IRs were consistently slightly higher with abatacept versus other b/ tsDMARDs across all databases. However, in the metaanalysis, a small but significant increase in the risk of total malignancy (HR [95\% CI] 1.09 [1.02-1.16]) associated with abatacept versus other b/tsDMARDs emerged. An evaluation of all malignancies was performed, and no one type of malignancy was found to be more prevalent in abatacept compared with other b/tsDMARDs initiators (Additional file 1: Table S10).

The regression analyses for a single diagnosis of prespecified malignancy types showed no significant difference in the abatacept versus other b/tsDMARDs initiators in any database, with one exception (Fig. 2). A small but significant increase in the risk of lung cancer with abatacept versus other $b / t s D M A R D s$ was identified in the PharMetrics database (HR [95\% CI] 1.62 [1.03-2.54]). The meta-analyses showed small, but not statistically significant, increases in the risk of lung cancer (Fig. 2b; HR [95\% CI] 1.10 [0.62-1.96]), lymphoma (Fig. 2c; 1.27 [0.941.72]), breast cancer (Fig. 2d; 1.15 [0.92-1.45]), and NMSC (Fig. 2e; 1.10 [0.93-1.30]) with abatacept versus other b/ tsDMARDs, consistent with that seen for the overall malignancy rate.

\section{Infection-related outcomes}

The IRs for hospitalized infections were slightly lower in the abatacept-treated patients compared with the $\mathrm{b} /$

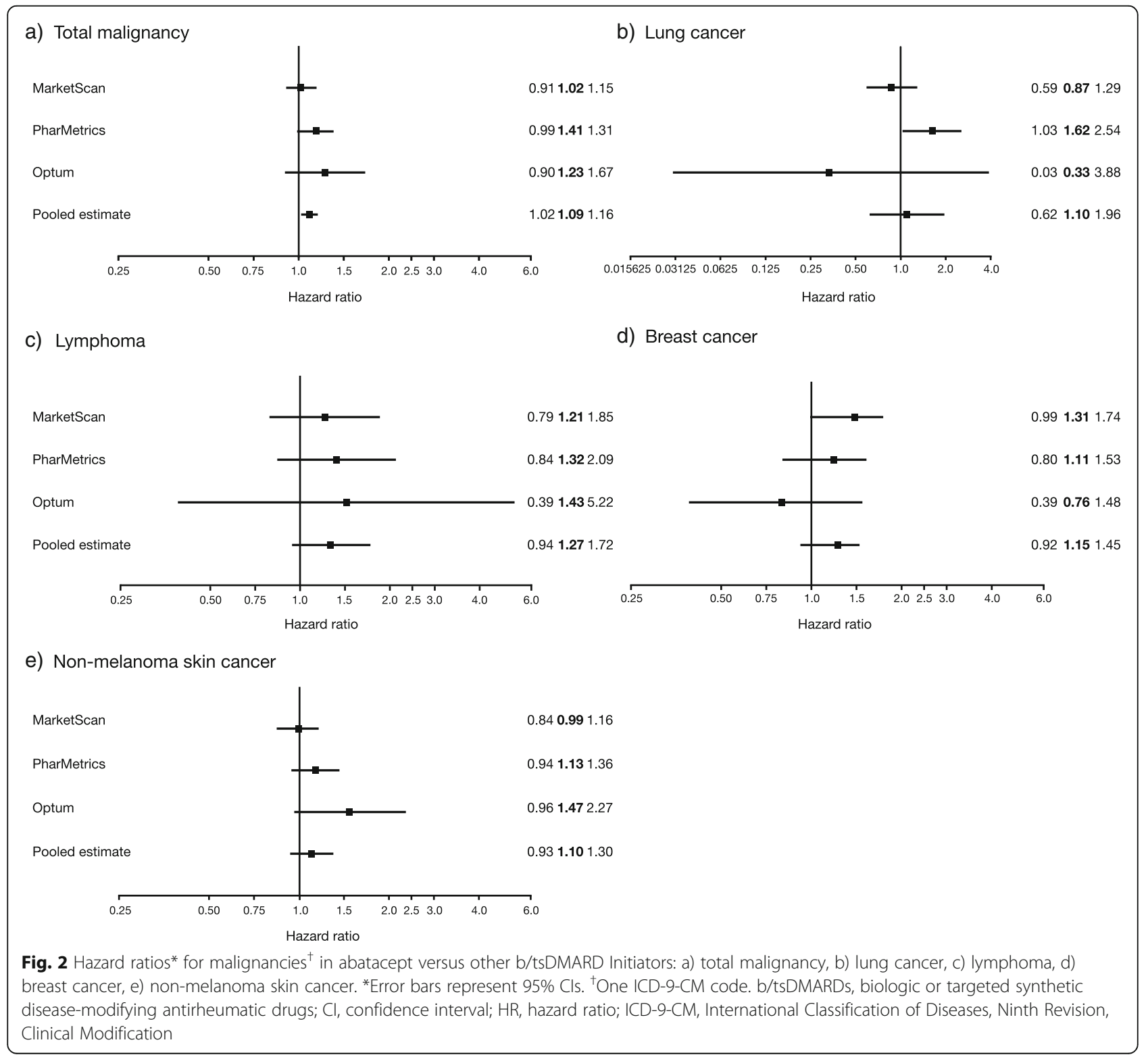


tsDMARDs group overall (Additional file 1: Table S11) and by age strata (Additional file 1: Table S12). The analyses of individual databases showed no significant difference in the risk of hospitalized infections in abatacept initiators compared with other b/tsDMARDs initiators (Fig. 3a); this finding was confirmed by the results from the meta-analysis (HR [95\% CI] 0.96 [0.84-1.09]).

For opportunistic infections, the regression analyses in individual databases and the meta-analysis (HR [95\% CI] 1.06 [0.96-1.17]) showed no significant difference in risk in abatacept initiators relative to b/tsDMARDs initiators (Fig. 3b). The IRs for opportunistic infections were slightly higher in the abatacept-treated patients compared with the $\mathrm{b} / \mathrm{tsDMARDs}$ group (Additional file 1: Table S11). In the sensitivity analysis requiring two ICD9-CM codes, a small but significantly increased opportunistic infection risk with abatacept was observed in the PharMetrics database (HR [95\% CI] 1.30 [1.07-1.58]). The results for the MarketScan and Optum databases (data not shown) were comparable with those of the primary analysis.

Applying the Calderwood algorithm [30] to the TB outcome analysis reduced the total number of events from 160, 104, and 40 to 10, 4, and 0 in the MarketScan, PharMetrics and Optum databases, respectively. Similar risk of TB was observed among abatacept and other b/ tsDMARDs initiators in the MarketScan (HR [95\% CI] 1.93 [0.45-8.32]) and PharMetrics (1.73 [0.17-17.63]) databases. It was not possible to analyze TB as an outcome in the Optum database because there were no events in the abatacept group and only two in the other b/tsDMARDs group. Thus, a meta-analysis of the outcome of TB was not performed. The IRs for tuberculosis varied across databases (Additional file 1: Table S11).

\section{Discussion}

In this real-world study of safety outcomes in patients with RA using data from three large US healthcare databases, a slight increase in the risk of total malignancy associated with abatacept versus other b/tsDMARD treatment was observed. Meta-analyses of specific malignancy types (lung cancer, lymphoma, breast cancer, or NMSC) showed similar trends, but the differences were not statistically significant. The risks of hospitalized infections, opportunistic infections, and TB were similar in patients receiving abatacept as in those receiving other b/tsDMARDs.

Evidence is inconsistent as to whether the use of abatacept, compared with other b/tsDMARDs, is associated with an increased risk of malignancy [27, 28, 34-36]. Contrary to the results of previous population-based cohort studies, which included evaluations of all NMSC [35] and squamous cell skin cancer [37], no significantly increased risk of NMSC was observed with abatacept compared with other b/tsDMARDs in this study. The results of this study are consistent with those from a meta-analysis of clinical trials that showed b/tsDMARDs, including abatacept, were not significantly associated with an increased malignancy risk compared with csDMARDs or placebo [36]. Previous interventional trials and real-world analyses of abatacept demonstrated a similar malignancy risk of abatacept compared with placebo or other comparators [27, 28, 34]. There may be several reasons for the small but statistically significantly increased risk of overall malignancy seen with abatacept treatment, including the unique upstream mechanism of action of abatacept and differences in patient characteristics. Abatacept inhibits the CD80/CD86:CD28 costimulatory signal required for full $\mathrm{T}$ cell activation and as a) Hospitalized infections

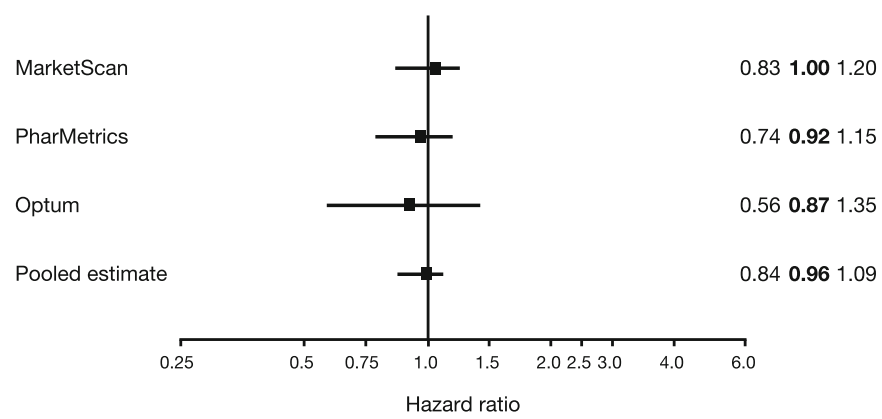

b) Opportunistic infections ${ }^{\ddagger}$

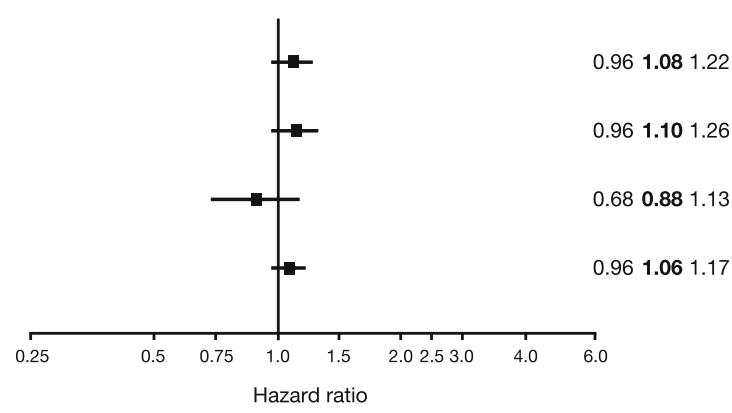

Fig. 3 Hazard ratios* for a) hospitalized infectionst and b) opportunistic infections in abatacept versus other b/tsDMARD initiators. Similar risk of TB was observed among abatacept and other b/tsDMARDs initiators in the MarketScan (HR [95\% CI] 1.93 [0.45-8.32]) and PharMetrics (1.73 [0.1717.63]) databases. It was not possible to analyze TB as an outcome in the Optum database because there were no events in the abatacept group and only two in the other b/tsDMARDs group. A meta-analysis of the outcome of TB was not performed due to low numbers. *Error bars represent $95 \% \mathrm{Cls}$. tData represent hospitalizations associated with an infection as the primary diagnosis (one ICD-9-CM code). b/tsDMARDs, biologic or targeted synthetic disease-modifying antirheumatic drugs; $\mathrm{Cl}$, confidence interval; HR, hazard ratio; ICD-9-CM, International Classification of Diseases, Ninth Revision, Clinical Modification; IR, incidence rate; TB, tuberculosis 
such may alter the immune responses to tumors, as well as dampening pathogenic autoimmunity [38]. The finding of increased total malignancy risk needs further investigation but should be considered with the following limitations in mind. There were some noticeable differences in baseline characteristics, such as age, sex, percentage of patients with other autoimmune diseases, and, particularly, prior exposure to b/tsDMARDs and csDMARDs between the abatacept and other b/ tsDMARDs groups, which may have had an impact on the malignancy outcome. Propensity score matching was used in our study to control for confounding factors; however, residual confounding from unmeasured factors, such as clinical characteristics or smoking history, could not be ruled out. Prior exposure to b/tsDMARDs is particularly important for residual confounding considerations, as the treatments included for exposure analysis were the same as those included in the comparator group (other b/tsDMARDs) for the outcome analysis. A higher percentage of patients had prior exposure to $\mathrm{b} /$ tsDMARDs in the abatacept (49-58\%) versus the comparator (14-19\%) group. It could be speculated that patients who initiated abatacept may be at a more advanced disease stage and therefore received a greater number of prior therapies, compared with those who initiated other b/tsDMARDs, which was not adequately controlled for in our analysis. Rather than matching on this differential timing of cohort entry, we adjusted for these imbalances, which may not have fully removed the bias from the imbalance in prior exposure to other b/ tsDMARDs [39]. This is particularly the case when attributing malignancies occurring after abatacept use to abatacept exposure as prior use of other b/tsDMARDs may also contribute to the occurrence of malignancies. In this study, a latency period of 180 days was used in an effort to address this challenge. Medications and comorbid conditions that may influence malignancy risk were assessed during the 180-day baseline period, but changes were not assessed during the follow-up period. In addition, the short latency period of 180 days and follow-up period of 747-970 days may be insufficient for the study of cancer.

An increased risk of opportunistic infections in patients with RA receiving tumor necrosis factor inhibitors compared with those receiving csDMARDs was observed in a systematic review of 87 articles and 40 abstracts and a real-world observational study of the Corrona registry $[40,41]$. However, limited comparative data are available for abatacept and other b/tsDMARDs [41]. This study suggests that risk of opportunistic infections is similar in patients receiving abatacept as in those receiving other b/tsDMARDs. In the sensitivity analysis, an elevated risk for opportunistic infection for abatacept was observed in one of the three databases. This observation may be due to the imbalances between the groups, such as greater co-medication differences in abatacept versus other b/ tsDMARDs initiators in the PharMetrics database compared with those in other databases (Additional file 1: Table S7). The similar hospitalized infection risk in this study is consistent with the findings from previous studies comparing b/tsDMARDs including abatacept with csDMARDs $[14,42]$ and when comparing abatacept with other b/tsDMARDs [43].

Previous research has shown that ICD-9-CM codes alone may not yield a satisfactory positive predictive value for TB identification [44]. Thus, a published algorithm [30] was applied to increase the specificity of the TB identification in this study. The risk of TB was similar in patients receiving abatacept as in those receiving other $\mathrm{b} /$ tsDMARDs; however, this was not statistically significant. The comparable risk for TB with abatacept and other $\mathrm{b} /$ tsDMARDs observed in our study is consistent with the previously reported low IRs for TB in patients treated with $\mathrm{b} / \mathrm{tsDMARDs}$, including abatacept, using data from clinical trials and national registries, when correct screening and prophylaxis was applied [27, 45]. The low event count in the Optum database and lack of sufficient data for metaanalysis in our study preclude further conclusion, and further assessment of TB risk in larger studies is warranted.

Further limitations of this study should be considered. Since treatment was determined from the prescribing or dispensing records in each database, adherence to any of the RA treatments cannot be confirmed. As with any observational study based on claims data, identification of medical events or baseline co-morbid conditions was limited to data that were captured as part of the claim. Furthermore, the data captured in the databases were not primarily collected for research purposes; thus, diagnostic codes and algorithms were utilized to identify outcomes but cannot serve as a confirmation of the outcome. Finally, as both biologic-experienced and biologic-naïve patients were analyzed together, due to latency, it was not possible to eliminate the influence of prior therapy on malignancy risk. Despite these limitations, the sample size and, therefore, the ability to detect potential effects were maximized in this study by including data from three large claims databases.

\section{Conclusions}

In this analysis of three large real-world US claims databases, abatacept initiators had a slightly increased risk of total malignancy compared with initiators of other b/ tsDMARDs. Meta-analyses showed similar, but not statistically significant, trends for lung cancer, lymphoma, breast cancer, and NMSC. However, due to the imbalance in prior b/tsDMARD use between the groups, it was not possible to rule out residual confounding despite statistical adjustment or to identify the potential effects of prior b/ tsDMARD therapy. The risks for hospitalized infections, 
opportunistic infections, and TB were similar in patients with RA who initiated abatacept and those who initiated other b/tsDMARDs. The findings of this analysis are consistent with the established safety profile of abatacept.

\section{Supplementary information}

Supplementary information accompanies this paper at https://doi.org/10. 1186/s13075-019-1992-x.

Additional file 1:. Statistical analyses: variable selection for models. Supplementary tables. (DOCX $39 \mathrm{~kb})$

\begin{abstract}
Abbreviations
b/tsDMARD: Biologic and targeted synthetic disease-modifying antirheumatic drug; Cl: Confidence interval; csDMARD: Conventional synthetic diseasemodifying antirheumatic drug; HR: Hazard ratio; ICD-9-CM: International Classification of Diseases, Ninth Revision, Clinical Modification; IR: Incidence rate; NMSC: Non-melanoma skin cancer; py: Person-years; RA: Rheumatoid arthritis; SD: Standard deviation; TB: Tuberculosis; TNF: Tumor necrosis factor
\end{abstract}

\section{Acknowledgements}

Professional medical writing and editorial assistance were provided by $\mathrm{Bu}$ Reinen, PhD, and Lola Parfitt, MRes, at Caudex and was funded by BristolMyers Squibb.

\section{Authors' contributions}

All authors have made substantial contributions to the conception or design of the work and acquisition, analysis, or interpretation of data for the work; drafted the work or revised it critically for important intellectual content; gave final approval of the version to be published; and agreed to be accountable for all aspects of the work in ensuring that questions related to the accuracy or integrity of any part of the work are appropriately investigated and resolved.

\section{Funding}

This study was sponsored by Bristol-Myers Squibb.

\section{Availability of data and materials}

Bristol-Myers Squibb policy on data sharing may be found at https://www. bms.com/researchers-and-partners/clinical-trials-and-research/disclosurecommitment.html.

\section{Ethics approval and consent to participate}

Not applicable

\section{Consent for publication}

Not applicable

\section{Competing interests}

TAS and NB are employees and shareholders of Bristol-Myers Squibb. MB is a consultant for Bristol-Myers Squibb and has received consulting fees $(<\$ 10,000)$ from Pfizer, UCB, and Teva in the past 2 years. MH is a shareholder of Theralogix LLC, has received grant/research support $(<\$ 10,000)$ from the National Institutes of Health, and is a consultant for Bristol-Myers Squibb, EMD Serono, Genentech/Roche, Novartis, Pfizer, and UCB. MLS and SSu are consultants to Bristol-Myers Squibb. NR and SSi are employees of Mu Sigma and have received research grants $(<\$ 10,000)$ from Bristol-Myers Squibb. AG-C is a shareholder of GlaxoSmithKline and Bristol-Myers Squibb and is an employee of Bristol-Myers Squibb.

\section{Author details}

${ }^{1}$ Bristol-Myers Squibb, Princeton, NJ, USA. ${ }^{2}$ Amsterdam University Medical Centers, Vrije Universiteit, Amsterdam, The Netherlands. ${ }^{3}$ University of Maryland School of Medicine, Baltimore, MD, USA. ${ }^{4}$ Mu Sigma, Bengaluru, Karnataka, India. ${ }^{5}$ McGill University, Montreal, Quebec, Canada.
Received: 15 January 2019 Accepted: 5 September 2019

Published online: 08 November 2019

\section{References}

1. Kvien TK. Epidemiology and burden of illness of rheumatoid arthritis. Pharmacoeconomics. 2004;22:1-12.

2. Shaw Y, Bradley M, Dominique A, Michaud K, McDonald D, Simon TA Responding resiliently to chronic disease: rheumatoid arthritis patients' discourse on coping strategies and challenges. Ann Rheum Dis. 2018;77: abstract FRI0740-HPR.

3. Shaw Y, Zhang C, Schumacher R, McDonald D, Simon TA, Michaud K. Understanding the burden of rheumatoid arthritis using qualitative research: which impacts are not captured by patient-reported measures? Ann Rheum Dis. 2018;77:abstract FRI0725-HPR.

4. Zhang C, Shaw Y, Shakley B, Ferri L, Michaud K, McDonald D, et al. Understanding fatigue burden and coping strategies in rheumatoid arthritis using qualitative research. Ann Rheum Dis. 2018;77:abstract AB1444-HPR.

5. Doran MF, Crowson CS, Pond GR, O'Fallon WM, Gabriel SE. Frequency of infection in patients with rheumatoid arthritis compared with controls: a population-based study. Arthritis Rheum. 2002;46:2287-93.

6. Smitten AL, Choi HK, Hochberg MC, Suissa S, Simon TA, Testa MA, et al. The risk of hospitalized infection in patients with rheumatoid arthritis. J Rheumatol. 2008;35:387-93.

7. Smitten AL, Simon TA, Hochberg MC, Suissa S. A meta-analysis of the incidence of malignancy in adult patients with rheumatoid arthritis. Arthritis Res Ther. 2008;10:R45.

8. Simon TA, Thompson A, Gandhi KK, Hochberg M, Suissa S. Incidence of malignancy in adult patients with rheumatoid arthritis: a meta-analysis. Arthritis Res Ther. 2015;17:212.

9. Zintzaras E, Voulgarelis M, Moutsopoulos HM. The risk of lymphoma development in autoimmune diseases: a meta-analysis. Arch Intern Med. 2005;165:2337-44

10. Bongartz T, Sutton AJ, Sweeting MJ, Buchan I, Matteson EL, Montori V. AntiTNF antibody therapy in rheumatoid arthritis and the risk of serious infections and malignancies: systematic review and meta-analysis of rare harmful effects in randomized controlled trials. JAMA. 2006;295:2275-85.

11. Listing J, Strangfeld A, Kary S, Rau R, von Hinueber U, Stoyanova-Scholz M, et al. Infections in patients with rheumatoid arthritis treated with biologic agents. Arthritis Rheum. 2005:52:3403-12.

12. Leombruno JP, Einarson TR, Keystone EC. The safety of anti-tumour necrosis factor treatments in rheumatoid arthritis: meta and exposure-adjusted pooled analyses of serious adverse events. Ann Rheum Dis. 2009;68:1136-45.

13. Schneeweiss S, Setoguchi S, Weinblatt ME, Katz JN, Avorn J, Sax PE, et al. Anti-tumor necrosis factor alpha therapy and the risk of serious bacterial infections in elderly patients with rheumatoid arthritis. Arthritis Rheum. 2007:56:1754-64.

14. Simon TA, Askling J, Lacaille D, Franklin J, Wolfe F, Covucci A, et al. Infections requiring hospitalization in the abatacept clinical development program: an epidemiological assessment. Arthritis Res Ther. 2010;12:R67.

15. Solomon DH, Lunt M, Schneeweiss S. The risk of infection associated with tumor necrosis factor alpha antagonists: making sense of epidemiologic evidence. Arthritis Rheum. 2008:58:919-28.

16. Askling J, Fahrbach K, Nordstrom B, Ross S, Schmid CH, Symmons D. Cancer risk with tumor necrosis factor alpha (TNF) inhibitors: metaanalysis of randomized controlled trials of adalimumab, etanercept, and infliximab using patient level data. Pharmacoepidermiol Drug Saf. 2011 . 20:119-30.

17. Schiff M, Weinblatt ME, Valente R, van der Heijde D, Citera G, Elegbe A, et al. Head-to-head comparison of subcutaneous abatacept versus adalimumab for rheumatoid arthritis: two-year efficacy and safety findings from AMPLE trial. Ann Rheum Dis. 2014;73:86-94.

18. Ramiro S, Sepriano A, Chatzidionysiou K, Nam JL, Smolen JS, van der Heijde D, et al. Safety of synthetic and biological DMARDs: a systematic literature review informing the 2016 update of the EULAR recommendations for management of rheumatoid arthritis. Ann Rheum Dis. 2017:76:1101-36.

19. Bedi B, Li JY, Grassi F, Tawfeek H, Weitzmann MN, Pacifici R. Inhibition of antigen presentation and T cell costimulation blocks PTH-induced bone loss. Ann N Y Acad Sci. 2010;1192:215-21.

20. Bingham CO III. Emerging therapeutics for rheumatoid arthritis. Bull NYU Hosp Jt Dis. 2008;66:210-5. 
21. Cutolo M, Nadler S. Advances in CTLA-4-lg-mediated modulation of inflammatory cell and immune response activation in rheumatoid arthritis. Autoimmun Rev. 2013;12:758-67.

22. Davis PM, Nadler SG, Stetsko DK, Suchard SJ. Abatacept modulates human dendritic cell-stimulated T-cell proliferation and effector function independent of IDO induction. Clin Immunol. 2008:126:38-47.

23. Alten R, Kaine J, Keystone E, Nash P, Delaet I, Qi K, et al. Safety of SC abatacept in patients with RA: update from pooled clinical trial data. Ann Rheum Dis. 2012;71:670

24. Alten R, Kaine J, Keystone EC, Nash P, Delaet I, Qi K, et al. Safety of subcutaneous abatacept in patients with rheumatoid arthritis (RA): integrated analysis of five clinical trials up to 4.5 years. Ann Rheum Dis. 2011;70(Suppl 3):617.

25. Becker JC, Westhovens R, Hochberg M, Qi K, Kelly S, Smitten A, et al. The long-term safety of abatacept in the treatment of rheumatoid arthritis: integrated analyses from the abatacept clinical trial program. Ann Rheum Dis. 2010;69(Suppl3):377.

26. Escudero Contreras A, Castro-Villegas MC, Hernández-Hernández MV, DíazGonzález F. Efficacy and safety of abatacept in patients with rheumatoid arthritis and no prior treatment with biologics. Reumatol Clin. 2011;7:392-6.

27. Alten R, Kaine J, Keystone E, Nash P, Delaet I, Genovese MC. Long-term safety of subcutaneous abatacept in rheumatoid arthritis: integrated analysis of clinical trial data representing more than four years of treatment. Arthritis Rheumatol. 2014;66:1987-97.

28. Simon TA, Smitten AL, Franklin J, Askling J, Lacaille D, Wolfe F, et al. Malignancies in the rheumatoid arthritis abatacept clinical development programme: an epidemiological assessment. Ann Rheum Dis. 2009;68:1819-26.

29. MacLean CH, Park GS, Traina SB, Liu HH, Hahn BH, Paulus HE, et al. Positive predictive value (PPV) of an administrative data-based algorithm for the identification of patients with rheumatoid arthritis (RA). Arthritis Rheum. 2001;44:S106.

30. Calderwood MS, Platt R, Hou X, Malenfant J, Haney G, Kruskal B, et al. Real-time surveillance for tuberculosis using electronic health record data from an ambulatory practice in eastern Massachusetts. Public Health Rep. 2010;125:843-50.

31. Berlin JA. The use of meta-analysis in pharmacoepidemiology. In: Strom BL, editor. Pharmacoepidemiology. 3rd Editon ed. West Sussex: Wiley; 2000. p. 633-659.

32. DerSimonian R, Laird N. Meta-analysis in clinical trials. Control Clin Trials. 1986;7:177-88.

33. Higgins JP, Thompson SG. Quantifying heterogeneity in a meta-analysis. Stat Med. 2002;21:1539-58.

34. Kim SC, Pawar A, Desai RJ, Solomon DH, Gale S, Bao M, et al. Risk of malignancy associated with use of tocilizumab versus other biologics in patients with rheumatoid arthritis: a multi-database cohort study. Semin Arthritis Rheum. 2019 Mar 9. https://doi.org/10.1016/j.semarthrit.2019.03.002 [Epub ahead of print].

35. Montastruc F, Renoux C, Dell'Aniello S, Simon TA, Azoulay L, Hudson M, et al. Abatacept initiation in rheumatoid arthritis and the risk of cancer: a population-based comparative cohort study. Rheumatology. 2019;58:683-91.

36. Lopez-Olivo MA, Tayar JH, Martinez-Lopez JA, Pollono EN, Cueto JP, Gonzales-Crespo MR, et al. Risk of malignancies in patients with rheumatoid arthritis treated with biologic therapy: a meta-analysis JAMA. 2012;308:898-908.

37. Wadström H, Frisell T, Askling J, ARTIS Study Group. Malignant neoplasms in patients with rheumatoid arthritis treated with tumor necrosis factor inhibitors, tocilizumab, abatacept, or rituximab in clinical practice: a nationwide cohort study from Sweden. JAMA Intern Med. 2017;177:1605-12

38. Herrero-Beaumont G, Martinez Calatrava MJ, Castaneda S. Abatacept mechanism of action: concordance with its clinical profile. Reumatol Clin. 2012:8:78-83.

39. Suissa S, Moodie EE, Dell'Aniello S. Prevalent new-user cohort designs for comparative drug effect studies by time-conditional propensity scores. Pharmacoepidermiol Drug Saf. 2017;26:459-68.

40. Greenberg JD, Reed G, Kremer JM, Tindall E, Kavanaugh A, Zheng C, et al. Association of methotrexate and tumour necrosis factor antagonists with risk of infectious outcomes including opportunistic infections in the CORRONA registry. Ann Rheum Dis. 2010;69:380-6.

41. Nam JL, Winthrop KL, van Vollenhoven RF, Pavelka K, Valesini G, Hensor EM, et al. Current evidence for the management of rheumatoid arthritis with biological disease-modifying antirheumatic drugs: a systematic literature review informing the EULAR recommendations for the management of RA. Ann Rheum Dis. 2010:69:976-86.

42. Dixon WG, Watson $K$, Lunt M, Hyrich KL, Silman AJ, Symmons DP, et al. Rates of serious infection, including site-specific and bacterial intracellular infection, in rheumatoid arthritis patients receiving anti-tumor necrosis factor therapy: results from the British Society for Rheumatology Biologics Register. Arthritis Rheum. 2006;54:2368-76.

43. Mori S, Yoshitama T, Hidaka T, Sakai F, Hasegawa M, Hashiba Y, et al. Comparative risk of hospitalized infection between biological agents in rheumatoid arthritis patients: a multicenter retrospective cohort study in Japan. PLoS One. 2017:12:e0179179.

44. Barber C, Lacaille D, Fortin PR. Systematic review of validation studies of the use of administrative data to identify serious infections. Arthritis Care Res. 2013;65:1343-57.

45. Cantini F, Niccoli L, Goletti D. Tuberculosis risk in patients treated with nonanti-tumor necrosis factor-a (TNF-a) targeted biologics and recently licensed TNF-a inhibitors: data from clinical trials and national registries. J Rheumatol Suppl. 2014;91:56-64

\section{Publisher's Note}

Springer Nature remains neutral with regard to jurisdictional claims in published maps and institutional affiliations.
Ready to submit your research? Choose BMC and benefit from:

- fast, convenient online submission

- thorough peer review by experienced researchers in your field

- rapid publication on acceptance

- support for research data, including large and complex data types

- gold Open Access which fosters wider collaboration and increased citations

- maximum visibility for your research: over $100 \mathrm{M}$ website views per year

At BMC, research is always in progress.

Learn more biomedcentral.com/submissions 